\title{
ERRATUM: "FERMI LARGE AREA TELESCOPE STUDY OF COSMIC-RAYS AND THE INTERSTELLAR MEDIUM IN NEARBY MOLECULAR CLOUDS” (2012, ApJ, 755, 22)
}

M. Ackermann ${ }^{1}$, M. Ajello ${ }^{2}$, A. Allafort ${ }^{2}$, L. Baldini ${ }^{3}$, J. Ballet ${ }^{4}$, G. Barbiellini ${ }^{5,6}$, D. Bastieri ${ }^{7,8}$, K. BeChtol $^{2}$,

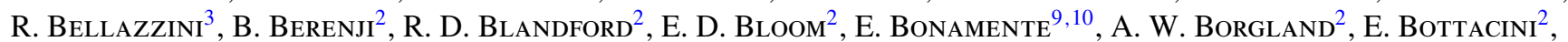
T. J. Brandt ${ }^{11,12}$, J. Bregeon ${ }^{3}$, M. Brigida ${ }^{13,14}$, P. Bruel ${ }^{15}$, R. Buehler ${ }^{2}$, G. Busetto ${ }^{7,8}$, S. Buson ${ }^{7,8}$, G. A. Caliandro ${ }^{16}$, R. A. Cameron ${ }^{2}$, P. A. Caraveo ${ }^{17}$, J. M. Casandian ${ }^{4}$, C. Cecchi ${ }^{9}, 10$, E. Charles ${ }^{2}$, A. Chekhtman ${ }^{18,63}$, J. Chiang ${ }^{2}$, S. Ciprini ${ }^{10,19}$, R. Claus ${ }^{2}$, J. Cohen-Tanugi ${ }^{20}$, J. Conrad ${ }^{21,22,64}$, F. D’Ammando ${ }^{9,23,24}$, A. De Angelis ${ }^{25}$, F. De Palma ${ }^{13,14}$, C. D. Dermer ${ }^{26}$, S. W. Digel ${ }^{2}$, E. Do Couto e Silva ${ }^{2}$, P. S. Drell ${ }^{2}$, A. Drlica-Wagner ${ }^{2}$, L. Fallettio ${ }^{20}$, C. Favuzzi ${ }^{13,14}$, S. J. Fegan ${ }^{15}$, E. C. Ferrara ${ }^{27}$, W. B. Focke ${ }^{2}$, Y. FukaZawa ${ }^{28}$, Y. Fukui ${ }^{29}$, S. Funk ${ }^{2}$, P. Fusco ${ }^{13}, 14$, F. Gargano ${ }^{14}$, D. Gasparrini ${ }^{30}$, S. Germani ${ }^{9,10}$, N. Giglietto ${ }^{13,14}$, F. Giordano ${ }^{13,14}$, M. Giroletti ${ }^{31}$, T. Glanzman ${ }^{2}$, G. Godfrey ${ }^{2}$,

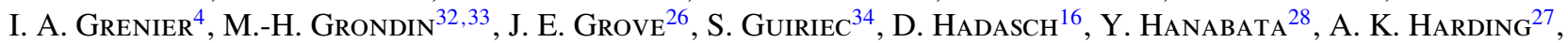

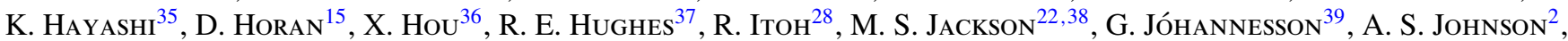
T. KAMAE ${ }^{2}$, H. KATAGiRI ${ }^{40}$, J. KATAOKA ${ }^{41}$, J. KNÖDLSEDER ${ }^{11,12}$, M. Kuss ${ }^{3}$, J. LANDE ${ }^{2}$, S. LARsson ${ }^{21,22,42}$, S.-H. LeE ${ }^{43}$,

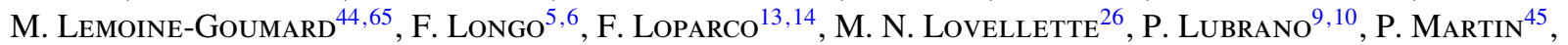
M. N. Mazziotta ${ }^{14}$, J. E. McEneri ${ }^{27,46}$, J. Mehault ${ }^{20}$, P. F. Michelson ${ }^{2}$, W. Mitthumsiri ${ }^{2}$, T. Mizuno ${ }^{28}$, A. A. Moiseev ${ }^{46,47}$, C. Monte ${ }^{13,14}$, M. E. Monzani ${ }^{2}$, A. Morsellit ${ }^{48}$, I. V. Moskalenko ${ }^{2}$, S. Murgia ${ }^{2}$, M. Naumann-Godo ${ }^{4}$,

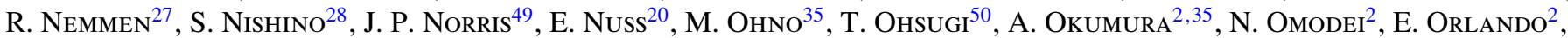
J. F. Ormes $^{51}$, M. Ozaki ${ }^{35}$, D. Paneque ${ }^{2,52}$, J. H. Panetta ${ }^{2}$, D. Parent ${ }^{18,63}$, M. Pesce-Rollins ${ }^{3}$, M. Pierbattista ${ }^{4}$, F. Piron ${ }^{20}$,

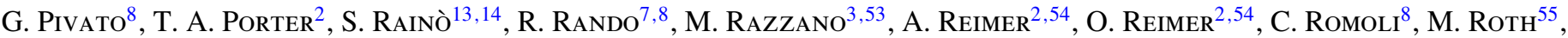
T. SAda ${ }^{28}$, H. F.-W. SAdrozinski ${ }^{53}$, D. A. SANChez ${ }^{32}$, C. Sbarra ${ }^{7}$, C. SGrò ${ }^{3}$, E. J. Siskind ${ }^{56}$, G. Spandre ${ }^{3}$, P. SPineldi ${ }^{13,14}$, A. W. Strong ${ }^{45}$, D. J. Suson ${ }^{57}$, H. TAKahashi ${ }^{50}$, T. TAKahashi ${ }^{35}$, T. TANAKA ${ }^{2}$, J. G. Thayer ${ }^{2}$, J. B. Thayer ${ }^{2}$,

D. J. Thompson ${ }^{27}$, L. Tibaldo ${ }^{7,8}$, O. Tibolla ${ }^{58}$, M. Tinivella ${ }^{3}$, D. F. Torres ${ }^{16,59}$, G. Tosti ${ }^{9,10}$, A. TramaCere ${ }^{2,60,61}$,

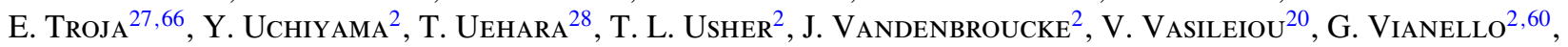

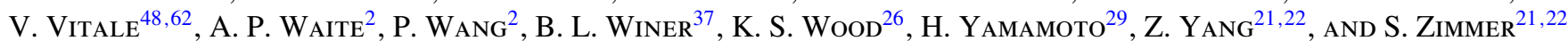
${ }^{1}$ Deutsches Elektronen Synchrotron DESY, D-15738 Zeuthen, Germany

${ }^{2}$ W. W. Hansen Experimental Physics Laboratory, Kavli Institute for Particle Astrophysics and Cosmology, Department of Physics and SLAC National Accelerator Laboratory, Stanford University, Stanford, CA 94305, USA

${ }^{3}$ Istituto Nazionale di Fisica Nucleare, Sezione di Pisa, I-56127 Pisa, Italy

${ }^{4}$ Laboratoire AIM, CEA-IRFU/CNRS/Université Paris Diderot, Service d'Astrophysique, CEA Saclay, 91191 Gif sur Yvette, France

${ }^{5}$ Istituto Nazionale di Fisica Nucleare, Sezione di Trieste, I-34127 Trieste, Italy

${ }^{6}$ Dipartimento di Fisica, Università di Trieste, I-34127 Trieste, Italy

${ }^{7}$ Istituto Nazionale di Fisica Nucleare, Sezione di Padova, I-35131 Padova, Italy

${ }^{8}$ Dipartimento di Fisica “G. Galilei,” Università di Padova, I-35131 Padova, Italy

${ }_{9}^{9}$ Istituto Nazionale di Fisica Nucleare, Sezione di Perugia, I-06123 Perugia, Italy

${ }^{10}$ Dipartimento di Fisica, Università degli Studi di Perugia, I-06123 Perugia, Italy

${ }^{11}$ CNRS, IRAP, F-31028 Toulouse cedex 4, France

12 GAHEC, Université de Toulouse, UPS-OMP, IRAP, Toulouse, France

${ }^{13}$ Dipartimento di Fisica “M. Merlin” dell'Università e del Politecnico di Bari, I-70126 Bari, Italy

${ }^{14}$ Istituto Nazionale di Fisica Nucleare, Sezione di Bari, 70126 Bari, Italy

${ }^{15}$ Laboratoire Leprince-Ringuet, École polytechnique, CNRS/IN2P3, Palaiseau, France

${ }^{16}$ Institut de Ciències de l'Espai (IEEE-CSIC), Campus UAB, 08193 Barcelona, Spain

${ }^{17}$ INAF-Istituto di Astrofisica Spaziale e Fisica Cosmica, I-20133 Milano, Italy

${ }^{18}$ Center for Earth Observing and Space Research, College of Science, George Mason University, Fairfax, VA 22030, USA 19 ASI Science Data Center, I-00044 Frascati (Roma), Italy

${ }^{20}$ Laboratoire Univers et Particules de Montpellier, Université Montpellier 2, CNRS/IN2P3, Montpellier, France

${ }^{21}$ Department of Physics, Stockholm University, AlbaNova, SE-106 91 Stockholm, Sweden

22 The Oskar Klein Centre for Cosmoparticle Physics, AlbaNova, SE-106 91 Stockholm, Sweden ${ }^{23}$ IASF Palermo, 90146 Palermo, Italy

${ }^{24}$ INAF-Istituto di Astrofisica Spaziale e Fisica Cosmica, I-00133 Roma, Italy

${ }^{25}$ Dipartimento di Fisica, Università di Udine and Istituto Nazionale di Fisica Nucleare, Sezione di Trieste, Gruppo Collegato di Udine, I-33100 Udine, Italy

${ }^{26}$ Space Science Division, Naval Research Laboratory, Washington, DC 20375-5352, USA

${ }^{27}$ NASA Goddard Space Flight Center, Greenbelt, MD 20771, USA

${ }^{28}$ Department of Physical Sciences, Hiroshima University, Higashi-Hiroshima, Hiroshima 739-8526, Japan; mizuno@ hirax6.hepl.hiroshima-u.ac.jp

${ }^{29}$ Department of Physics and Astrophysics, Nagoya University, Chikusa-ku Nagoya 464-8602, Japan

${ }^{30}$ Agenzia Spaziale Italiana (ASI) Science Data Center, I-00044 Frascati (Roma), Italy

${ }^{31}$ INAF Istituto di Radioastronomia, 40129 Bologna, Italy

${ }^{32}$ Max-Planck-Institut für Kernphysik, D-69029 Heidelberg, Germany

${ }^{33}$ Landessternwarte, Universität Heidelberg, Königstuhl, D 69117 Heidelberg, Germany

${ }^{34}$ Center for Space Plasma and Aeronomic Research (CSPAR), University of Alabama in Huntsville, Huntsville, AL 35899, USA

35 Institute of Space and Astronautical Science, JAXA, 3-1-1 Yoshinodai, Chuo-ku, Sagamihara, Kanagawa 252-5210, Japan; khayashi@ astro.isas.jaxa.jp

${ }^{36}$ Centre d'Études Nucléaires de Bordeaux Gradignan, IN2P3/CNRS, Université Bordeaux 1, BP120, F-33175 Gradignan Cedex, France

${ }^{37}$ Department of Physics, Center for Cosmology and Astro-Particle Physics, The Ohio State University, Columbus, OH 43210, USA

${ }^{38}$ Department of Physics, Royal Institute of Technology (KTH), AlbaNova, SE-106 91 Stockholm, Sweden

${ }^{39}$ Science Institute, University of Iceland, IS-107 Reykjavik, Iceland

${ }^{40}$ College of Science, Ibaraki University, 2-1-1, Bunkyo, Mito 310-8512, Japan 


\author{
${ }^{41}$ Research Institute for Science and Engineering, Waseda University, 3-4-1, Okubo, Shinjuku, Tokyo 169-8555, Japan \\ 42 Department of Astronomy, Stockholm University, SE-106 91 Stockholm, Sweden \\ ${ }^{43}$ Yukawa Institute for Theoretical Physics, Kyoto University, Kitashirakawa Oiwake-cho, Sakyo-ku, Kyoto 606-8502, Japan \\ ${ }^{44}$ Université Bordeaux 1, CNRS/IN2p3, Centre d'Études Nucléaires de Bordeaux Gradignan, 33175 Gradignan, France \\ ${ }^{45}$ Max-Planck Institut für extraterrestrische Physik, 85748 Garching, Germany \\ ${ }^{46}$ Department of Physics and Department of Astronomy, University of Maryland, College Park, MD 20742, USA \\ ${ }^{47}$ Center for Research and Exploration in Space Science and Technology (CRESST) and NASA Goddard Space Flight Center, Greenbelt, MD 20771, USA \\ 48 Istituto Nazionale di Fisica Nucleare, Sezione di Roma “Tor Vergata," I-00133 Roma, Italy \\ ${ }^{49}$ Department of Physics, Boise State University, Boise, ID 83725, USA \\ ${ }^{50}$ Hiroshima Astrophysical Science Center, Hiroshima University, Higashi-Hiroshima, Hiroshima 739-8526, Japan \\ ${ }^{51}$ Department of Physics and Astronomy, University of Denver, Denver, CO 80208, USA \\ 52 Max-Planck-Institut für Physik, D-80805 München, Germany \\ ${ }^{53}$ Santa Cruz Institute for Particle Physics, Department of Physics and Department of Astronomy and Astrophysics, \\ University of California at Santa Cruz, Santa Cruz, CA 95064, USA \\ ${ }^{54}$ Institut für Astro- und Teilchenphysik and Institut für Theoretische Physik, Leopold-Franzens-Universität Innsbruck, A-6020 Innsbruck, Austria \\ ${ }^{55}$ Department of Physics, University of Washington, Seattle, WA 98195-1560, USA \\ ${ }^{56}$ NYCB Real-Time Computing Inc., Lattingtown, NY 11560-1025, USA \\ ${ }^{57}$ Department of Chemistry and Physics, Purdue University Calumet, Hammond, IN 46323-2094, USA \\ ${ }^{58}$ Institut für Theoretische Physik and Astrophysik, Universität Würzburg, D-97074 Würzburg, Germany \\ ${ }^{59}$ Institució Catalana de Recerca i Estudis Avançats (ICREA), Barcelona, Spain \\ ${ }^{60}$ Consorzio Interuniversitario per la Fisica Spaziale (CIFS), I-10133 Torino, Italy \\ ${ }^{61}$ INTEGRAL Science Data Centre, CH-1290 Versoix, Switzerland \\ 62 Dipartimento di Fisica, Università di Roma “Tor Vergata," I-00133 Roma, Italy \\ Received 2013 September 23; published 2013 November 6
}

Online-only material: color figures

In the published version of the paper, errors were made in calculating the exposure time due to an analysis mistake. While they do not affect gas emissivities of the R CrA and Cepheus \& Polaris flare regions significantly (the differences are within the systematic uncertainty), that of the Chamaeleon region is increased by $\sim 20 \%$. Although we claimed a difference of $\sim 50 \%$ in gas emissivity among these molecular cloud regions in the original paper, it is decreased to $\sim 30 \%$ (comparable to the sum of the statistical and systematic uncertainties) in the revised analysis. Therefore, our conclusion of the original paper, that a small variation $(\sim 20 \%)$ of the CR density in the solar neighborhood exists, is not supported by the data if we take these uncertainties into account. On the other hand, the obtained $X_{\mathrm{CO}}$ and $X_{\mathrm{Av}}$ values, and the masses of gas calculated from them are not changed significantly (the differences are within the statistical errors). Errors and corrections in the original paper are summarized below.

1. In the Abstract (lines 5-6) and Section 3 (lines 4-5 in the 3rd paragraph) in the original paper, the $\gamma$-ray emissivity

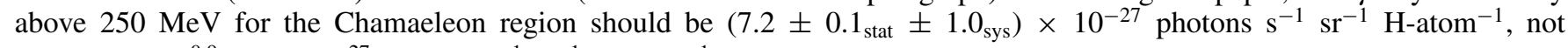
$\left(5.9 \pm 0.1_{\text {stat }}{ }_{-1.0}^{+0.9}\right.$ sys $) \times 10^{-27}$ photons s$^{-1} \mathrm{sr}^{-1} \mathrm{H}$-atom ${ }^{-1}$.

2. In the Abstract (lines 8-10), "Whereas the energy dependences of the emissivities agree well with that predicted from direct CR observations at the Earth, the measured emissivities from $250 \mathrm{MeV}$ to $10 \mathrm{GeV}$ indicate a variation of the CR density by $20 \%$ in the neighborhood of the solar system, even if we consider the systematic uncertainties." should be changed to "The energy dependences of the emissivities agree well with that predicted from direct CR observations at the Earth. Although the measured emissivities from $250 \mathrm{MeV}$ to $10 \mathrm{GeV}$ differ by $\sim 30 \%$ among these molecular cloud regions, the difference is not significant if we take the systematic uncertainty into account."

3. Table 1 and Figure 13, which show gas emissivities and spectra for the Chamaeleon region in the original paper, should be changed to the Table 1 and Figure 1 as shown below.

4. Figure 16, which compares $\mathrm{H}_{\mathrm{I}}$ gas emissivities among several regions in the original paper, should be changed to Figure 2 as shown below.

5. The text from the line 13 to the last one in the first paragraph of Section 4.1, "The spectral shapes for the three regions..., indicating a difference of the CR density between the Chamaeleon and the others as shown in Figure 16." should be changed to the paragraph that follows.

"The shaded area of each spectrum indicates the systematic uncertainty as described in Section 3 . We note that the systematic uncertainty of the LAT effective area (5\% at $100 \mathrm{MeV}$ and $20 \%$ at $10 \mathrm{GeV}$; Rando et al. 2009) does not affect the relative value of emissivities. The effect of unresolved point sources is small; we have verified that the obtained emissivities are almost unaffected by decreasing the threshold for point sources from TS $=100$ to $\mathrm{TS}=50$. We also confirmed that the residual excess of photons around $\left(l=280^{\circ}\right.$ to $288^{\circ}, b=-20^{\circ}$ to $-12^{\circ}$; see the bottom panel of Figure 8$)$ in the Chamaeleon region does not affect the local H i emissivity very much. Thus the total systematic uncertainty is reasonably expressed by the shaded area shown in

\footnotetext{
${ }_{63}^{63}$ Resident at Naval Research Laboratory, Washington, DC 20375, USA.

${ }_{65}^{64}$ Royal Swedish Academy of Sciences Research Fellow, funded by a grant from the K. A. Wallenberg Foundation.

${ }^{65}$ Funded by contract ERC-StG-259391 from the European Community.

${ }^{66}$ NASA Postdoctoral Program Fellow, USA.
} 

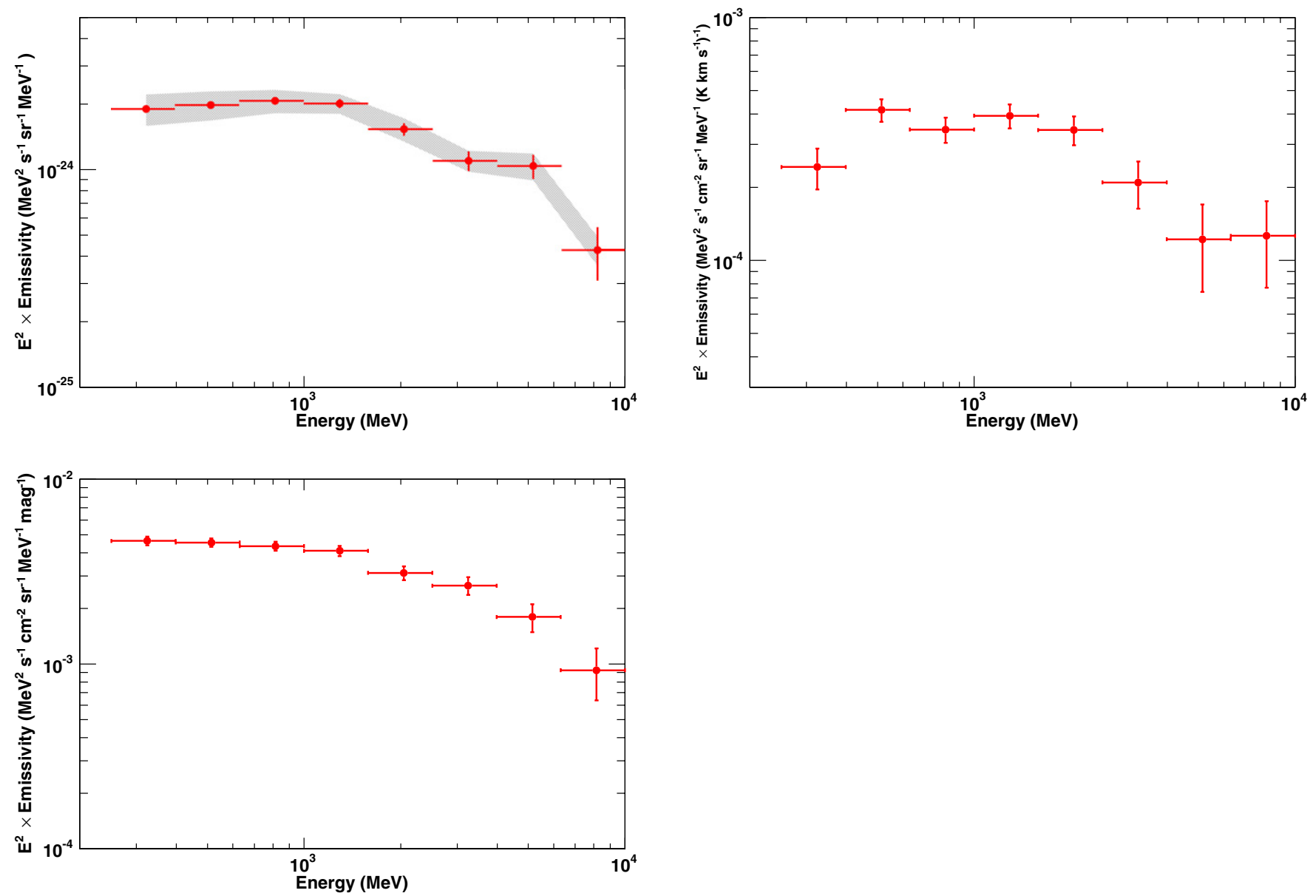

Figure 1. Emissivity spectrum of the local H I gas (top left), that per $W_{\mathrm{CO}}$ unit (top right), and that per unit $A v_{\text {res }}$ magnitude (bottom left) of the Chamaeleon region. The shaded area shows systematic uncertainties for H I (see the text for details).

(A color version of this figure is available in the online journal.)

Table 1

Gas Emissivities in the Chamaeleon Region with Their Statistical Uncertainties (Assuming $T_{\mathrm{S}}=125 \mathrm{~K}$ for the H I Maps Preparation)

\begin{tabular}{lccc}
\hline \hline $\begin{array}{l}\text { Energy } \\
(\mathrm{GeV})\end{array}$ & $q_{\mathrm{H}, 1}$ & $q_{\mathrm{CO}}$ & $q_{\mathrm{Av}}$ \\
\hline $0.25-0.40$ & $2.8 \pm 0.1$ & $0.35 \pm 0.07$ & $0.68 \pm 0.04$ \\
$0.40-0.63$ & $1.83 \pm 0.07$ & $0.39 \pm 0.04$ & $0.42 \pm 0.02$ \\
$0.63-1.00$ & $1.21 \pm 0.05$ & $0.20 \pm 0.02$ & $0.25 \pm 0.01$ \\
$1.00-1.58$ & $0.74 \pm 0.03$ & $0.15 \pm 0.02$ & $0.15 \pm 0.01$ \\
$1.58-2.51$ & $0.36 \pm 0.02$ & $0.08 \pm 0.01$ & $0.073 \pm 0.006$ \\
$2.51-3.98$ & $0.16 \pm 0.02$ & $0.031 \pm 0.006$ & $0.039 \pm 0.004$ \\
$3.98-6.31$ & $0.10 \pm 0.01$ & $0.011 \pm 0.004$ & $0.017 \pm 0.003$ \\
$6.31-10.00$ & $0.025 \pm 0.007$ & $0.008 \pm 0.003$ & $0.006 \pm 0.002$ \\
Total & $7.2 \pm 0.1$ & $1.22 \pm 0.09$ & $1.64 \pm 0.05$ \\
\hline
\end{tabular}

Notes. Units; $q_{\mathrm{H}, 1}\left(10^{-27} \mathrm{~s}^{-1} \mathrm{sr}^{-1}\right), q_{\mathrm{CO}}\left(10^{-6} \mathrm{~cm}^{-2} \mathrm{~s}^{-1} \mathrm{sr}^{-1}\left(\mathrm{~K} \mathrm{~km} \mathrm{~s}^{-1}\right)^{-1}\right)$, $q_{\mathrm{Av}}\left(10^{-5} \mathrm{~cm}^{-2} \mathrm{~s}^{-1} \mathrm{sr}^{-1} \mathrm{mag}^{-1}\right)$.

Figure 2. The spectral shapes for the three regions agree well with the LIS models, indicating that the CR nuclei have similar spectral distribution in the vicinity of the solar system. On the other hand, the apparent difference of emissivities among the three regions (by $\sim 30 \%$ ) is comparable to the uncertainty (statistical and systematic errors). Therefore the LAT data do not allow us to claim or exclude a possible small variation of the CR density in the solar neighborhood."

6. In Section 5 (lines 4-9 in the 2nd paragraph), "The emissivities, however, indicate a variation of the CR density by $\sim 20 \%$ within $\sim 300 \mathrm{pc}$ around the solar system, even if we consider the systematic uncertainties. We consider possible origins of the variation are non-uniform supernova rate and anisotropy of CRs depending on the propagation conditions" should be changed to "Although the emissivities differ by $\sim 30 \%$ among these regions, the difference is not significant if we take the systematic uncertainty into account. If the variation really exists, possible origins of it might be non-uniform supernova rate and anisotropy of CRs, depending on the propagation conditions." 


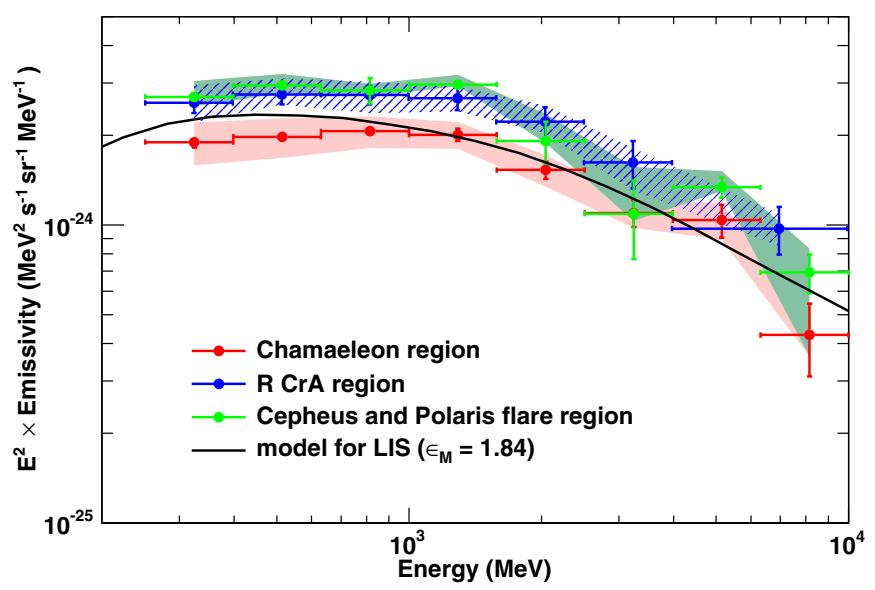

Figure 2. H I emissivity spectra of the Chamaeleon, R CrA, and Cepheus \& Polaris flare regions compared with the model for the LIS with the nuclear enhancement factor of 1.84. The shaded areas for the Chamaeleon, R CrA, and Cepheus and Polaris flare spectra indicate the systematic uncertainty evaluated in Section 3.

(A color version of this figure is available in the online journal.)

\section{REFERENCE}

Rando, R., \& the Fermi LAT Collaboration 2009, arXiv:0907.0626 